\title{
Cognitive functioning following traumatic brain injury: A five-year follow-up
}

\author{
Nigel V. Marsh ${ }^{\mathrm{a}, *}$, Maria R. Ludbrook ${ }^{\mathrm{b}}$ and Lauren C. Gaffaney ${ }^{\mathrm{b}}$ \\ ${ }^{a}$ Department of Psychology, Sunway University, Bandar Sunway, Selangor, Malaysia \\ ${ }^{\mathrm{b}}$ Department of Psychology, University of Waikato, Hamilton, New Zealand
}

\begin{abstract}
.
OBJECTIVE: To describe the long-term prevalence and severity of cognitive deficits following significant (i.e., ventilation required for $>24$ hours) traumatic brain injury. To assess a comprehensive range of cognitive functions using psychometric measures with established normative, reliability, and validity data.

METHODS: A group of 71 adults was assessed at approximately five years (mean $=66$ months) following injury. Assessment of cognitive functioning covered the domains of intelligence, attention, verbal and visual memory, visual-spatial construction, and executive functions.

RESULTS: Impairment was evident across all domains but prevalence varied both within and between domains. Across aspects of intelligence clinical impairment ranged from $8-25 \%$, attention $39-62 \%$, verbal memory $16-46 \%$, visual memory $23-51 \%$, visual-spatial construction 38\%, and executive functions (verbal fluency) $13 \%$. In addition, 3-23\% of performances across the measures were in the borderline range, suggesting a high prevalence of subclinical deficit.

CONCLUSIONS: Although the prevalence of impairment may vary across cognitive domains, long-term follow-up documented deficits in all six domains. These findings provide further evidence that while improvement of cognitive functioning following significant traumatic brain injury may be possible, recovery of function is unlikely.
\end{abstract}

Keywords: Traumatic brain injury, cognitive functioning, neuropsychological assessment, long-term outcome

\section{Introduction}

Traumatic brain injury (TBI) is the most common cause of disability for young adults in Western industrialised countries (Lezak, Howieson, \& Loring, 2004). The consequences of TBI can be life-long and affect multiple aspects of an individual's quality of life (Masel \& DeWitt, 2010). TBI can result in impairment in physical, cognitive, and psychosocial functioning but it is the impairment in cognitive functioning that has the greatest impact on functional outcomes such as employment and basic daily living skills (Rassovsky et al., 2006). Age at injury and severity of injury are two important predictors of the degree of long-term impairment following TBI. Younger age at injury is associated with less longterm cognitive impairment (Marsh \& Whitehead

\footnotetext{
*Address for correspondence: Nigel V. Marsh, Department of Psychology, Sunway University, No. 5 Jalan Universiti, Bandar Sunway, 47500 Selangor Darul Ehsan, Malaysia. Tel.: +6 037491 8622; Fax: +6 035635 8633; E-mail: nigelm@sunway.edu.my.
}

2005; Senathi-Raja, Ponsford, \& Schonberger, 2010) and the more severe the injury the greater the degree of long-term cognitive impairment and associated disability (Dikmen, Machamer, Powell, \& Temkin, 2003; Rohling, Meyers, \& Millis, 2003).

Despite evidence of improvement in cognitive functioning over the first year following TBI (Kersel, Marsh, Havill, \& Sleigh, 2001), there is also considerable evidence to indicate that in TBI of at least moderate severity full recovery of cognitive abilities rarely occurs (Dikmen, Corrigan, Levin, Machamer, Stiers, \& Weisskopf, 2009). Cognitive deficits remain evident for many years following the initial injury and make a major contribution to the long-term level of overall disability (Draper \& Ponsford, 2008; Ruttan, Martin, Liu, Colella, \& Green, 2008; Whitnall, McMillan, Murray, \& Teasdale, 2006).

Assessment of cognitive functioning has evolved over the last 75 years from consideration of global constructs, such as intelligence, to a focus on specific domains of cognitive functioning (Kersel et al., 
2001). These domains were initially conceptualised from observations of behavioural functioning. They have been further developed through theoretical advances in the understanding of the possible components of the cognitive processes that underlie overt behaviour. Despite these developments there is also evidence that these hypothesised constructs may overlap (Clune-Ryberg et al., 2011). However consideration of cognitive functioning from a domain perspective continues to provide useful insights into improving rehabilitation practices and understanding the impact of cognitive impairment on real-world functioning and quality of life (DeLuca, Schultheis, Madigan, Christodoulou, \& Averill, 2000; Silverberg $\&$ Millis, 2009).

The cognitive domain of attention incorporates speed of information processing, working memory, and sustained attention. Assessment of people with TBI has consistently found impairment in all aspects of attention (Kennedy, Clement, \& Curtiss, 2003; Slovarp, Azuma, \& LaPointe, 2012). Similarly impairment in most aspects of both verbal and visual memory functioning is also commonly reported following TBI (Vakil, 2005; Ashton, Donders, \& Hoffman, 2005; Schwarz, Penna, \& Novack, 2009). Impairment in the domain of visual perception is less commonly assessed for in TBI although there is some evidence for impairment in the constructional and organisational skills aspects (McKenna, Cooke, Fleming, Jefferson, \& Ogden, 2006). Executive function is perhaps the most multifaceted domain of cognitive functioning and the aspect of verbal fluency has been consistently shown to demonstrate impairment following TBI (Henry \& Crawford, 2004).

While there are now a number of studies that provide information on cognitive functioning following TBI across a range of cognitive domains, the majority of these studies use follow up periods of one or two years (Dikmen et al., 2009). Given that TBI can have life-long affects on multiple aspects of a person's quality of life (Masel \& DeWitt, 2010), it is important to develop a research base on the long-term effects on cognitive functioning. A search of the literature found only three studies over the last 25 years that systematically examined cognitive functioning 4-6 years following TBI.

Tate, Fenelon, Manning, and Hunter (1991) examined patterns of neuropsychological impairment in 85 adults assessed, on average, at six years following TBI. The three main cognitive domains examined in this study were basic neuropsychological skills, speed of information processing, and learning and memory. Residual deficits were common with $70 \%$ of subjects demonstrating clinically significant deficits. The greatest prevalence of impairment was in memory and learning (57\%), followed by speed of information processing (34\%).

Johnstone, Hexum, and Ashkanazi (1995) reported on the extent of impairment in specific cognitive functions, relative to estimates of premorbid functioning, for 97 adults at approximately four years following TBI. Intelligence was the cognitive domain that showed the least decline, followed by attention, memory, and then speed of information processing. The greatest decline was evident in the cognitive domain of executive functions, specifically cognitive flexibility.

Millis et al. (2001) presented the results from a comprehensive assessment of cognitive functioning at, on average, five years following TBI. Their sample was of people who had received predominately moderate to severe TBI. The sample size varied across the measures ranging from 141 to 182 subjects. Of particular note was the wide variability in outcome on the measures of cognitive functioning, ranging from no measureable impairment to severe impairment. Cognitive deficits were most frequent on measures of verbal memory (50\% significant impairment) and attention (33-56\% significant impairment across different measures).

The aim of this study was to describe the longterm prevalence and severity of cognitive deficits following significant (i.e., ventilation required for $>24$ hours) TBI in a clearly defined adult sample. The literature base in this area consists of only a few studies and these studies do not necessarily assess a wide range of cognitive functions. In this study assessment of a comprehensive range of cognitive abilities was undertaken using established psychometric measures with proven utility in the assessment of TBI. Ongoing cognitive deficits have a major impact on the longterm level of disability following TBI (Rassovsky et al., 2006). Therefore the information obtained in this study may usefully inform rehabilitation professionals when they are advising patients and family caregivers on the future options for the person with significant TBI.

\section{Method}

\subsection{Subjects}

The subjects for this study were selected from those people enrolled in the Waikato Traumatic Brain 
Table 1

Demographic and clinical information for the traumatic brain injury patients $(n=71)$

\begin{tabular}{lc}
\hline Gender & \\
Female & $19(27 \%)$ \\
Male & $52(73 \%)$ \\
Age at follow-up (years) & \\
Mean & 31 \\
Standard deviation & 12 \\
Range & $16-62$ \\
Years of Education & \\
Mean & 12 \\
Standard deviation & 2 \\
Range & $8-18$ \\
Cause of TBI & \\
Road traffic crash & $55(78 \%)$ \\
Fall & $7(10 \%)$ \\
Assault & $5(7 \%)$ \\
Other & $4(6 \%)$ \\
Glasgow Coma Scale category (on admission) & \\
Severe injury & $55(78 \%)$ \\
Moderate injury & $10(14 \%)$ \\
Mild injury & $6(9 \%)$ \\
Months since TBI & \\
Mean & 66 \\
Standard deviation & 9 \\
Range & $50-91$ \\
Glasgow Outcome Scale (at follow-up) & \\
Severe disability & $14(20 \%)$ \\
Moderate disability & $18(25 \%)$ \\
Good recovery & $39(55 \%)$ \\
\hline
\end{tabular}

Injury Study (Havill, Sleigh, Kersel, \& Marsh, 1998). The criterion for admission to the study was that the patient was required to be ventilated on clinical grounds for $>24$ hours where ventilation was, at least in part, required for treatment of the TBI. Exclusion criteria were non-English speaking and previous hospital admission for TBI or psychiatric illness.

Five year outcome data was obtained for 104(85\%) of the original sample of 123 patients. Of these 29 (28\%) were deceased, the majority having died as a result of their TBI. Of the surviving 75 patients, 4 $(5 \%)$ were less than 16 years of age at the time of the five-year follow-up. The remaining 71 adult patients constitute the sample reported on here. Severity of TBI was determined by Glasgow Coma Scale score (Teasdale \& Jennett, 1974) on admission to hospital. Overall outcome at five years was assessed by means of the Glasgow Outcome Scale (Jennett \& Bond, 1975). The results from these two clinical variables and demographic information on the sample are presented in Table 1.

\subsection{Measures}

Subjects were administered a comprehensive battery of psychometric tests and self-report ques- tionnaires to assess different aspects of their neuropsychological functioning. This report details the results from the six measures of cognitive functioning that were administered. These psychometric tests assessed the six cognitive domains of intelligence, attention, verbal and visual memory, visual-spatial construction, and executive functions.

Intelligence was assessed by administration of the Wechsler Adult Intelligence Scale - Third edition (WAIS-III; Wechsler, 1997). The WAIS-III and it earlier versions are the most frequently used measures in neuropsychological assessment (Strauss, Sherman, $\&$ Spreen, 2006). Attention was assessed by administration of the Symbol Digit Modalities Test (SDMT; Smith, 1982) and the Trail Making Test (TMT; Tombaugh, 2004). The SDMT is extremely sensitive to the presence of cognitive impairment in both adults and children (Strauss et al., 2006). The TMT has a high sensitivity to the presence of cognitive impairment across different neuropsychological conditions (Mitrushina, Boone, Razani, \& D’Elia, 2005). Verbal memory was assessed by administration of the Rey Auditory-Verbal Learning Test (RAVLT; Schmidt, 1996). The RAVLT has been used extensively to assess memory functioning in both normal groups and a wide variety of clinical samples (Mitrushina et al., 2005). Visual memory was assessed by administration of the Rey Complex Figure Test (RCFT; Meyers \& Meyers, 1995). Visual-spatial constructional ability was assessed by administration of the copy trial of the RCFT. The RCFT consists of a complex twodimensional line drawing containing 18 details and is frequently used to assess visuospatial constructional and visual memory deficits in a wide variety of clinical groups (Mitrushina et al., 2005). Finally executive functions, specifically phonemic verbal fluency, were assessed by administration of the Controlled Oral Word Association test (COWA; Tombaugh, Kozak, \& Rees, 1999). The COWA evaluates the spontaneous production of words under restricted search conditions and has been found to be sensitive to impairment in executive processes in a wide variety of clinical groups including TBI (Strauss et al., 2006).

\subsection{Procedure and data analysis}

Ethical approval for the study was obtained from the Waikato Ethics Committee. Patients who had participated in either of the previous six month or one year follow-ups were re-contacted and asked to participate in a further five year follow-up. Those who agreed provided informed consent and were inter- 
viewed and assessed by postgraduate trainee clinical psychologists. Results from the six month and one year assessments have been reported previously for the 65 patients who completed both assessments (Kersel et al., 2001)

The order in which the cognitive tests were presented was the same for each TBI patient and was as follows: RCFT (copy \& immediate recall trials), WAIS-III (up to Block Design subtest), RCFT (delayed recall \& recognition trials), RAVLT, WAISIII (remaining subtests), RAVLT (delayed recall \& recognition trials), SDMT (written $\&$ oral versions), TMT (parts A \& B), and COWA test. All tests were administered and scored in accordance with the standardised instructions available for each measure.

Descriptive statistics are reported for the demographic and assessment data. Following established conventions in determining the clinical significance of individual scores (Mitrushina et al., 2005; Strauss et al., 2006), obtained scores from the 2 nd to the 8th percentiles were considered to indicate borderline performance and scores less than the 2 nd percentile were considered to indicate impaired performance. For the TMT where higher scores indicate greater impairment, scores from the 92nd to the 98th percentiles were considered to indicate borderline performance and scores greater than the 98th percentile were considered to indicate impaired performance.

\section{Results}

As reflected in the Glasgow Outcome Scale scores (Table 1), some individuals still had severe physical or cognitive impairments even five years after their TBI. Therefore some individuals were unable to complete certain tests. The number of completed assessments ranged from 67 for the RAVLT and COWA to 62 for the RCFT Recognition trial.

On the measure of general intelligence the following average scores were obtained: WAIS-III Full Scale IQ $=92.94(S D=19.03$, range $=56-132$, $n=66)$, WAIS-III Verbal Scale IQ $=93.79(S D=$ 19.41, range $=54-138, \quad n=66$ ), WAIS-III Performance IQ $=93.05(S D=17.33$, range $=59-127$, $n=66$ ), WAIS-III Verbal Comprehension Index $=$ 93.56 $(S D=19.68$, range $=55-142, n=66)$, WAIS-III Perceptual Organization Index $=100.14(S D=18.98$, range $=65-135, n=66$ ), WAIS-III Working Memory Index $=93.02(S D=18.82$, range $=50-126, n=66)$, and WAIS-III Processing Speed Index $=83.71$ $(S D=15.24$, range $=57-111, n=65)$. The prevalence
Table 2

Prevalence and severity of impairment on the Wechsler Adult Intelligence Scale - Third edition at five years following traumatic brain injury

\begin{tabular}{lccc}
\hline & \multicolumn{3}{c}{ Classification Level } \\
\cline { 2 - 4 } & No Impairment & Borderline & Impaired \\
\hline Verbal IQ & $49(74 \%)$ & $9(14 \%)$ & $8(12 \%)$ \\
Performance IQ & $49(74 \%)$ & $10(15 \%)$ & $7(11 \%)$ \\
Full Scale IQ & $52(79 \%)$ & $5(8 \%)$ & $9(14 \%)$ \\
$\quad$ Verbal Comprehension & $50(76 \%)$ & $11(17 \%)$ & $5(8 \%)$ \\
$\quad$ Index & & & \\
Perceptual Organization & $56(85 \%)$ & $5(8 \%)$ & $5(8 \%)$ \\
$\quad$ Index & & & \\
Working Memory Index & $52(79 \%)$ & $3(5 \%)$ & $11(17 \%)$ \\
Processing Speed Index & $40(62 \%)$ & $9(14 \%)$ & $16(25 \%)$ \\
\hline Bory
\end{tabular}

Borderline impairment $=2$ nd -8 th percentile, Impaired $<2$ nd percentile.

and severity of impairment on the WAIS-III is presented in Table 2.

On the measures of attention the following average scores were obtained: SDMT written $=$ $36.62(S D=14.06$, range $=6-63, n=66)$, SDMT oral $=42.40(S D=15.81$, range $=4-76, n=65)$, TMT part $A=49.29$ seconds $(S D=35.56$, range $=16-201$, $n=65$ ), and TMT part $B=118.78$ seconds ( $S D=$ 79.50 , range $=36-404, n=63$ ).

On the measure of verbal memory the following average scores were obtained: RAVLT trial 1 (immediate recall $)=5.16(S D=2.94$, range $=0-14, n=67)$, RVALT sum $=39.51(S D=15.43$, range $=4-72, n=$ 67), RAVLT retention $=7.55 \quad(S D=4.10$, range $=$ $0-15, \quad n=67), \quad$ RAVLT delayed recall $=6.75$ $(S D=4.52$, range $=0-15, n=67)$, and RAVLT recognition $=10.60 \quad(S D=3.79, \quad$ range $=1-15$, $n=65)$.

On the measure of visual memory the average scores obtained were: RCFT immediate recall $=$ $13.87(S D=8.50$, range $=0-30.5, n=63)$, RCFT delayed recall $=13.56(S D=8.56$, range $=0-29.5$, $n=63)$, and RCFT recognition $=19.42(S D=2.28$, range $=13-23, n=62$ ).

On the measure of visual-spatial construction, the RCFT copy trial, the average score was 29.92 $(S D=5.83$, range $=11.5-36.0, n=63)$. On the measure of verbal fluency, the COWA, the average score was $31.31(S D=12.68$, range $=2-60, n=67)$. The prevalence and severity of impairment on the five cognitive domains is presented in Table 3.

\section{Discussion}

The purpose of this study was to document the prevalence and severity of impairment in cognitive 
Table 3

Prevalence and severity of impairment across five domains of cognitive functioning at five years following traumatic brain injury

\begin{tabular}{|c|c|c|c|}
\hline \multirow[b]{2}{*}{ Domain } & \multicolumn{3}{|c|}{ Classification Level } \\
\hline & No Impairment & Borderline & Impaired \\
\hline \multicolumn{4}{|c|}{$\begin{array}{l}\text { Attention (Symbol Digit Modalities Test) } \\
\text { (S) }\end{array}$} \\
\hline Written score & $25(38 \%)$ & $13(20 \%)$ & $28(42 \%)$ \\
\hline Oral score & $31(47 \%)$ & $9(14 \%)$ & $26(39 \%)$ \\
\hline \multicolumn{4}{|c|}{ Attention (Trail Making Test) } \\
\hline Part A & $35(54 \%)$ & $5(8 \%)$ & $25(39 \%)$ \\
\hline Part B & $23(35 \%)$ & $2(3 \%)$ & $40(62 \%)$ \\
\hline \multicolumn{4}{|c|}{ Verbal Memory (Rey Auditory Verbal Learning Test) } \\
\hline Immediate recall & $42(63 \%)$ & $14(21 \%)$ & $11(16 \%)$ \\
\hline Total learning & $26(39 \%)$ & $11(16 \%)$ & $30(45 \%)$ \\
\hline Retention & $37(55 \%)$ & $7(10 \%)$ & $23(34 \%)$ \\
\hline Delayed recall & $30(45 \%)$ & $11(16 \%)$ & $26(39 \%)$ \\
\hline Recognition & $29(45 \%)$ & $6(9 \%)$ & $30(46 \%)$ \\
\hline \multicolumn{4}{|c|}{ Visual Memory (Rey Complex Figure Test) } \\
\hline Immediate recall & $26(41 \%)$ & $6(10 \%)$ & $31(49 \%)$ \\
\hline Delayed recall & $24(38 \%)$ & $7(11 \%)$ & $32(51 \%)$ \\
\hline Recognition & $34(55 \%)$ & $14(23 \%)$ & $14(23 \%)$ \\
\hline \multicolumn{4}{|c|}{ Visual-spatial Construction (Rey Complex Figure Test) } \\
\hline Copy & $29(46 \%)$ & $10(16 \%)$ & $24(38 \%)$ \\
\hline \multicolumn{4}{|c|}{ Executive Functions (Controlled Oral Word Association) } \\
\hline Total & $46(69 \%)$ & $12(18 \%)$ & $9(13 \%)$ \\
\hline
\end{tabular}

Borderline impairment $=2$ nd-8th percentile, Impaired $<2$ nd percentile. Except for Trail Making Test where Borderline impairment $=92 \mathrm{nd}-$ 98th percentile, Impaired $>98$ th percentile.

functioning in adults at five years following a significant TBI. The injuries were deemed to be significant because the patients required ventilation for greater than 24 hours following their injuries. Consistent with this criterion, the Glasgow Coma Scale scores for the sample indicated that $78 \%$ had suffered a severe TBI. Further, the fact that only $55 \%$ were rated as having made a 'good recovery' on the Glasgow Outcome Scale provides additional evidence of the significance of the patients' TBIs. Consistent with previous epidemiological findings with significant TBI (e.g., Lezak et al., 2004), the sample was predominately younger males who had obtained their TBI through road traffic crashes.

Interpretation of the patients' performance on the measures of cognitive functioning was undertaken by comparison to appropriate normative data. Although such comparisons can also be made using a sample of matched control subjects, normative samples are usually much larger giving greater confidence in the reliability of the results from the comparisons. Matched controls are primarily used to take into account any impact on performance of demographic variables such as age, gender, and years of education. However the large normative samples used in this study provided subgroup norms which allowed for any possible impact of such variables to be taken into account. Also, interpretation of the patients' performance with reference to normative data rather than data from matched controls is more consistent with usual clinical practice.

In terms of global and omnibus measures of cognitive functioning $79 \%$ of the patients were unimpaired on the Full Scale IQ score. This finding was reasonably consistent across the subcomponents of global cognitive functioning with $76 \%, 85 \%$, and $79 \%$ being unimpaired on the WAIS-III Verbal Comprehension, Perceptual Organization, and Working Memory indexes, respectively. However on the Processing Speed index only $62 \%$ were unimpaired suggesting that long-term the cognitive functions assessed by these tasks are relatively more impacted by TBI. This finding of comparatively less long-term impairment in IQ is consistent with previous findings (Johnstone et al., 1995). Similarly, the finding of $62 \%$ unimpaired on speed of information processing is consistent with the 66\% unimpaired reported by Tate et al. (1991).

Performance on the measures of attention showed a high prevalence of deficits with even more simple measures such as the oral score on the SDMT and Part A of the TMT showing 39\% of the sample with impaired performance. The prevalence of impairment increased considerably with increasing complexity of the measures and on the most complex measure of attention, Part B of the TMT, $62 \%$ of the sample was impaired. These two findings of both a high prevalence of impairment and an increase in the prevalence of impairment as a function of the 
complexity of the measure used were also reported by Millis et al. (2001) on their measures of attention.

On the measure of verbal memory $45 \%$ of the sample was impaired. This level of impairment is similar to that reported by other researchers who have conducted long-term follow-up studies of adult TBI patients (Millis et al., 2001; Tate et al., 1991). However not all aspects of verbal memory were equally impaired with the prevalence of impairment increasing as a function of time. Hence impairment was least evident in immediate memory, followed by retention, and delayed recall with the highest prevalence of impairment being in performance on the recognition trial (46\%). Unfortunately the high prevalence of impairment on the recognition trial suggests that a large number of people in the current sample have memory deficits due to problems with encoding. Therefore they may not benefit from the strategies based on retrieval that are commonly offered in memory rehabilitation programmes (Kersel et al., 2001).

On the measure of visual memory there was no evidence of a differential impact of time on memory performance. Approximately half of the sample was impaired on both the immediate and delayed trial. While visual memory has been assessed in other follow-up studies of adults with TBI (Ashton et al., 2005; Schwarz et al., 2009), these other studies were completed on samples that were in the early stages following injury. The prevalence of impairment found here suggests that assessment of visual memory should be included in long-term followup studies of TBI as approximately half the sample was still impaired at five years following injury. The prevalence of impairment in the recognition trial for visual memory was half that found for verbal memory. This suggests that there may be a subgroup of TBI patients who would benefit from memory rehabilitation strategies based on retrieval from visual memory even if they are unable to benefit when such strategies are focused on verbal memory.

On the measure of visual-spatial construction over a third $(38 \%)$ of the subjects were impaired. This domain of cognitive functioning is even less frequently researched in TBI outcome studies than visual memory. However there is some indication that researchers are beginning to incorporate this domain into their assessment protocols and one short-term follow-up study reported $26 \%$ of severe TBI patients to have deficits in constructional skills (McKenna et al., 2006). Given the fact that assessment of visual memory frequently requires the drawing of geometric shapes, assessment of the domain of visual-spatial construction is essential in order to ensure that performance on a visual memory measure is not confounded by impairment in visual-spatial constructional abilities (Ashton et al., 2005). In the current study performance on the copy trial was significantly related to performance on both the immediate $(r=0.64, n=63, p<0.001)$ and delayed $(r=0.63$, $n=63, p<0.001)$ recall trials of the RCFT.

Verbal fluency has been commonly viewed as a component of executive function (Mitrushina et al., 2005). In the current study $13 \%$ of the sample was impaired on this measure. This is less than the $18 \%$ reported by Millis et al. (2001). However they did use a more lenient cut-off of less than the 3 rd percentile to define impairment, and in the current study a further $18 \%$ of the sample was in the borderline range on the measure of verbal fluency. These results provide further support for the previously reported finding that verbal fluency is sensitive to the effects of TBI (Henry \& Crawford, 2004).

The results from the current study demonstrated the usefulness of taking a domain approach to the assessment of cognitive functioning. This allows for a better understanding of cognitive strengths and weaknesses than that obtained from solely assessing cognitive functioning as a global construct (e.g., intelligence). Such an approach also provides information on patient functioning in a form that is more useful to the planning of rehabilitation. However this does not mean that the domains are necessarily independent of each other (Clune-Ryberg et al., 2011; Schwarz et al., 2009). For example, the executive domain includes organisational and planning processes, and impairment in these aspects of cognition would impact on an individual's ability to draw a geometric shape, hence impacting on visual-spatial construction which would, in turn, impact on performance on a measure of visual memory. Hence assessment of cognitive functioning needs to be based on an understanding that while describing cognitive functions in terms of specific domains may be useful; multiple cognitive systems may be involved in any single behaviour of interest.

Overall the results from this study have demonstrated the wide range of impairment that is evident long-term across multiple domains of cognitive functioning following significant TBI. A limitation of this study is the cross-sectional nature of the design as this prevents an understanding of the temporal process of changes in cognitive functioning over time following TBI. Despite this limitation the current study is one of the few long-term studies available 
that has reported results for a clearly defined sample, across a wide range of cognitive functions. There is no basis to assume that the cognitive deficits frequently reported in studies of acute or short-term follow-up will be the same as those found in long-term followups. Hence, the results from the current study make a contribution to the development of a comprehensive understanding of long-term cognitive functioning following TBI.

Obtaining concurrent information on the functional abilities of people with TBI would add to the usefulness of studies on their cognitive ability. Future research should incorporate assessment of real world functioning along with a comprehensive assessment of cognitive functioning. This would allow researchers to establish the ecological validity of neuropsychological assessment thereby significantly increasing the utility of the process to the person with TBI and their family (Silverberg \& Millis, 2009). To develop a full understanding of the consequences of TBI as a process requires information on multiple aspects of patient functioning throughout their life span following the injury (Masel \& DeWitt, 2010).

\section{Acknowledgments}

This study was supported by grant number NF 98/17PG from the Neurological Foundation of New Zealand.

\section{Conflict of interest}

All of the authors declare that they have no conflict of interest.

\section{References}

Ashton, V. L., Donders, J., \& Hoffman, N. M. (2005). Rey Complex Figure Test performance after traumatic brain injury. Journal of Clinical and Experimental Neuropsychology, 27, 55-64.

Clune-Ryberg, M., Blanco-Campal, A., Carton, S., Pender, N., O’Brien, D., Phillips, J., Delargy, M., \& Burke, T. (2011). The contribution of retrospective memory, attention and executive functions to the prospective and retrospective components of prospective memory following TBI. Brain Injury, 25, 819-831.

DeLuca, J., Schultheis, M. T., Madigan, N. K., Christodoulou, C., \& Averill, A. (2000). Acquisition versus retrieval deficits in traumatic brain injury: Implications for memory rehabilitation. Archives of Physical Medicine and Rehabilitation, 81, 13271333.
Dikmen, S. S., Machamer, J. E., Powell, J. M., \& Temkin, N. R. (2003). Outcome 3 to 5 years after moderate to severe traumatic brain injury. Archives of Physical Medicine and Rehabilitation, 84, 1449-1457.

Dikmen, S. S., Corrigan, J. D., Levin, H. S., Machamer, J., Stiers, W., \& Weisskopf, M. G. (2009). Cognitive outcome following traumatic brain injury. Journal of Head Trauma Rehabilitation, 24, 430-438.

Draper, K., \& Ponsford, J. (2008). Cognitive functioning ten years following traumatic brain injury and rehabilitation. Neuropsychology, 22, 618-625.

Havill, J. H., Sleigh, J. W., Kersel, D. A., \& Marsh, N. V. (1998). Profile and cost of head injury patients admitted to the Waikato Hospital Intensive Care Unit. New Zealand Medical Journal, 111, 161-163.

Henry, J. D., \& Crawford, J. R. (2004). A meta-analytic review of verbal fluency performance in patients with traumatic brain injury. Neuropsychology, 18, 621-628.

Jennett, B., \& Bond, M. (1975). Assessment of outcome after severe brain damage: A practical scale. Lancet, 1, 480-484.

Johnstone, B., Hexum, C. L., \& Ashkanazi, G. (1995). Extent of cognitive decline in traumatic brain injury based on estimates of premorbid intelligence. Brain Injury, 9, 377-384.

Kennedy, J. E., Clement, P. F., \& Curtiss, G. (2003). WAIS-III Processing Speed Index scores after TBI: The influence of working memory, psychomotor speed and perceptual processing. The Clinical Neuropsychologist, 17, 303-307.

Kersel, D. A., Marsh, N. V., Havill, J. H., \& Sleigh, J. W. (2001). Neuropsychological functioning during the year following severe traumatic brain injury. Brain Injury, 15, 283-296.

Lezak, M. D., Howieson, D. B., \& Loring, D. W. (with Hannay, H. J., \& Fischer, J. S.). (2004). Neuropsychological assessment (4th ed.). New York: Oxford University Press.

Marsh, N. V., \& Whitehead, G. (2005). Skull fracture during infancy: A five-year follow-up. Journal of Clinical and Experimental Neuropsychology, 27, 352-366.

Masel, B. E., \& DeWitt, D. S. (2010). Traumatic brain injury: A disease process, not an event. Journal of Neurotrauma, 27, 1529-1540.

McKenna, K., Cooke, D. M., Fleming, J., Jefferson, A., \& Ogden, S. (2006). The incidence of visual perceptual impairment in patients with severe traumatic brain injury. Brain Injury, 20, 507-518.

Meyers, J. E., \& Meyers, K. R. (1995). Rey Complex Figure Test and recognition trial: Professional manual. Odessa, FL: Psychological Assessment Resources.

Millis, S. R., Rosenthal, M., Novack, T. A., Sherer, M., Nick, T. G., Kreutzer, J. S., High Jr., W. M., \& Ricker, J. H. (2001). Long-term neuropsychological outcome after traumatic brain injury. Journal of Head Trauma Rehabilitation, 16, 343-355.

Mitrushina, M., Boone, K. B., Razani, J., \& D'Elia, L. F. (2005). Handbook of normative data for neuropsychological assessment (2nd ed.). New York: Oxford University Press.

Rassovsky, Y., Satz, P., Alfano, M. S., Light, R. K., Zaucha, K., McArthur, D. L., \& Hovda, D. (2006). Functional outcome in TBI I: Neuropsychological, emotional, and behavioral mediators. Journal of Clinical and Experimental Neuropsychology, 28, 567-580.

Rohling, M. L., Meyers, J. E., \& Millis, S. R. (2003). Neuropsychological impairment following traumatic brain injury: A dose-response analysis. The Clinical Neuropsychologist, 17, 289-302. 
Ruttan, L., Martin, K., Liu, A., Colella, B., \& Green, R. E. (2008). Long-term cognitive outcome in moderate to severe traumatic brain injury: A meta-analysis examining timed and untimed tests at 1 and 4.5 or more years after injury. Archives of Physical Medicine and Rehabilitation, 89(Suppl 2), S69-S76.

Schmidt, M. (1996). Rey Auditory Verbal Learning Test: A handbook. Los Angeles: Western Psychological Services.

Schwarz, L., Penna, S., \& Novack, T. (2009). Factors contributing to performance on the Rey Complex Figure Test in individuals with traumatic brain injury. The Clinical Neuropsychologist, 23, 255-267.

Senathi-Raja, D., Ponsford, J., \& Schonberger, M. (2010). Impact of age on long-term cognitive function after traumatic brain injury. Neuropsychology, 24, 336-344.

Silverberg, N. D., \& Millis, S. R. (2009). Impairment versus deficiency in neuropsychological assessment: Implications for ecological validity. Journal of the International Neuropsychological Society, 15, 94-102.

Slovarp, L., Azuma, T., \& LaPointe, L. (2012). The effect of traumatic brain injury on sustained attention and working memory. Brain Injury, 26, 48-57.

Smith, A. (1982). Symbol Digit Modalities Test: Manual. Los Angeles, CA: Western Psychological Services.

Strauss, E., Sherman, E. M. S., \& Spreen, O. (2006). A compendium of neuropsychological tests: Administration, norms and commentary (3rd ed.). New York: Oxford University Press.
Tate, R. L., Fenelon, B., Manning, M. L., \& Hunter, M. (1991). Patterns of neuropsychological impairment after severe blunt head injury. The Journal of Nervous and Mental Disease, 179, 117-126.

Teasdale, G., \& Jennett, B. (1974). Assessment of coma and impaired consciousness: A practical scale. Lancet, 2, 81-84.

Tombaugh, T. N., Kozak, J., \& Rees, L. (1999). Normative data stratified by age and education for two measures of verbal fluency: FAS and animal naming. Archives of Clinical Neuropsychology, 14, 167-177.

Tombaugh, T. N. (2004). Trail Making Test A and B: Normative data stratified by age and education. Archives of Clinical Neuropsychology, 19, 203-214.

Vakil, E. (2005). The effect of moderate to severe traumatic brain injury (TBI) on different aspects of memory: A selective review. Journal of Clinical and Experimental Neuropsychology, 27, 977-1021.

Wechsler, D. (1997). Wechsler Adult Intelligence Scale - Third edition (WAIS-III): Administration and scoring manual. San Antonio, TX: Psychological Corporation.

Whitnall, L., McMillan, T. M., Murray, G. D., \& Teasdale, G. M. (2006). Disability in young people and adults after head injury: 5-7 year follow up of a prospective cohort study. Journal of Neurology, Neurosurgery, and Psychiatry, 77, 640-645. 